\title{
Using Gastrointestinal Distress Reports to Predict Youth Anxiety Risk: Implications for Mental Health Literacy and Community Care
}

Paul Alexander Bloom, $\mathrm{MA}^{1}$; Ian Douglas, $\mathrm{MS}^{2}$; Michelle VanTieghem, $\mathrm{PhD}^{1}$; Nim Tottenham, $\mathrm{PhD}^{1} ;$ \& Bridget Callaghan, $\mathrm{PhD}^{3}$

\author{
Affiliations: \\ ${ }^{1}$ Columbia University Department of Psychology \\ ${ }^{2}$ University of Texas at Austin Department of Psychology \\ ${ }^{3}$ University of California Los Angeles UCLA Department of Psychology \\ Address correspondence to: \\ Paul Alexander Bloom \\ Email:paul.bloom@columbia.edu
}

Short title: Youth GI symptoms and anxiety

\section{Acknowledgements:}

The authors would like to thank Andrea Fields, Tricia Choy, Nicolas Camacho, Lisa

Gibson, Anna Vannucci, and Nicole Gavrilova for their feedback and support in preparing this manuscript. The authors would also like to thank the Healthy Brain Network team for their efforts creating and managing open data repositories.

\section{Funding Statement:}

This work was supported by funding from the National Institutes of Mental Health (4R00MH113821) and the Brain \& Behavior Research Foundation (24739) for BC, the National Science Foundation (Graduate Research Fellowship) for PAB, and the National Institute of Mental Health (R01MH091864) and the Dana Foundation for NT.

\section{Conflicts of Interest: None}




\section{Abstract}

This study investigates the generalizability and predictive validity of associations between gastrointestinal (GI) symptoms and youth anxiety to establish

4 their utility in community mental health decision-making. We analyzed data from

5 youth ages 3-21 years in volunteer cohorts collected in Los Angeles $(\mathrm{N}=327)$ and New

6 York City $(\mathrm{N}=102)$, as well as the Healthy Brain Network cohort $(\mathrm{N}=1957)$. Youth

7 gastrointestinal distress was measured through items taken from the parent-reported

8 Child Behavior Checklist (CBCL). We examined generalizability of GI-anxiety

9 associations across cohorts and anxiety reporters, then evaluated the performance of

10 these models in predicting youth anxiety in holdout data. Consistent with previous

11 work, higher levels of gastrointestinal distress were associated with more parent-

12 reported youth anxiety behaviors in all three cohorts. Models trained on data from the

13 Healthy Brain Network cohort predicted parent-reported and child-reported anxiety

14 behaviors, as well as clinician-evaluated anxiety diagnoses, at above chance levels in

15 holdout data. Models which included gastrointestinal symptoms often, but not always,

16 outperformed models based on age and sex alone in predicting youth anxiety. Based

17 on the generalizability and predictive validity of GI-anxiety associations investigated

18 here, gastrointestinal symptoms may be an effective tool for child-facing professionals

19 for identifying children at risk for anxiety (Preprint: https://psyarxiv.com/zgavu/).

21 Keywords: gastrointestinal, anxiety, prediction, replication, youth 


\section{Introduction}

Psychiatric distress is increasingly common, especially among youth (Sakolsky \&

27 Birmaher, 2008; Twenge et al., 2019; Yatham et al., 2018). For example, the median age

28 of onset for anxiety disorders is 11 years (Kessler et al., 2005), and early onset mental

29 health problems are associated with more chronic courses of illness (Beesdo-Baum \&

30 Knappe, 2012; Godoy et al., 2019). As such, early intervention for mental health issues is

31 a public health concern, prompting several professional bodies to prioritize recognition,

32 referral and treatment competencies in the domain of youth mental health amongst their

33 members (Bell \& McKay, 2004).

34 Mental health care for youth is dispersed across multiple systems, including

35 family members, primary care providers (PCPs), schools (Luthar, Ebbert, et al., 2020;

36 Luthar, Kumar, et al., 2020; Moon et al., 2017), juvenile justice (Duong et al., 2020;

37 Loyd et al., 2019; Yoder et al., 2017), and child welfare (Gudiño et al., 2012; Shin,

38 2005). As PCPs are often the initial access point for mental health services, particularly

39 within economically disadvantaged communities, primary care is typically the first line of

40 defense for managing young people's mental health (Heneghan et al., 2008; O'Brien et

41 al., 2016). Over $25 \%$ of presentations to pediatric primary care are for psychosocial

42 distress (Cooper et al., 2006), and more presentations occur for unexplained physical and

43 somatic complaints that might be psychogenic (Rushton et al., 2002). Not surprisingly,

44 universal mental health screening has been recommended for PCPs, as well as teachers,

45 school nurses, and other child-facing professionals (Dowdy et al., 2015; Dvorsky et al.,

46 2014). Nonetheless, uptake of such screening is varied across contexts and is far from

47 universally adopted, even within pediatric primary care. Common concerns from teachers 
48 and primary care pediatricians include a lack of training and confidence in providing

49 treatments or making psychiatric referrals based on observed or reported psychological

50 states (Green et al., 2019; Reinke et al., 2011). Moreover, as many children may lack the

51 knowledge/insight to effectively report their mental health symptoms (Muris et al., 2010),

52 screening which relies only on self-reported psychological states may miss youth who

53 present with primarily physical manifestations of distress. Given such constraints, tools

54 that go beyond direct observation and understanding of psychological states are needed to

55 enhance detection of youth psychosocial distress.

One strategy to address limitations in professional expertise and child self-reports

57 of mental states is to use comorbid or prodromal physical health symptoms in treatment

58 and referral decisions. Mental health disorders are highly comorbid with somatic

59 symptoms (Bodas et al., 2008; Cohen et al., 1998; Koloski et al., 2012; Waters et al.,

60 2013), and gastrointestinal complaints (e.g., unexplained abdominal pain) in particular

61 are associated with concurrent and future anxiety in youth (Callaghan et al.,

62 undefined/ed; Campo et al., 2004; Kim et al., 2019; Saps et al., 2009), and are common

63 (8-10\%) in primary health settings (Starfield et al., 1980). Gastrointestinal distress

64 symptoms may thus be helpful in psychiatric referral processes for youth. Nonetheless, in

65 primary care settings, psychiatric referrals occur in less than $20 \%$ of gastrointestinal

66 distress cases even when a psychogenic root is suspected (Rushton et al., 2002),

67 suggesting there is room to improve the use of physical symptoms for psychiatric referral.

68 Considering that gastrointestinal symptoms are common and might be more easily

69 reported by youth, as well as observable to parents/PCPs/teachers/other child-facing

70 professionals, routine screening of gastrointestinal symptoms within primary care and 
71 community settings may assist individuals in making accurate treatment or referral

72 decisions for youth for mental health services, bolstering early intervention and

73 prevention efforts.

74 Previously (Callaghan et al., 2020), we demonstrated an association between

75 parent-reported child gastrointestinal distress and child anxiety using five questions

76 assessing gastrointestinal symptoms taken from the Child Behavior Checklist (CBCL;

77 Achenbach \& Edelbrock, 1983). In the current study, rather than investigating causal

78 mechanisms behind this relationship, we seek to generalize GI-anxiety associations

79 across samples and anxiety outcomes, and evaluate their potential for clinical use. If

80 responses on those items could be used in a predictive framework to identify youth at risk

81 for anxiety, this would highlight an opportunity for primary care and community settings

82 to incorporate reports of gastrointestinal symptoms into mental health screening and

83 referral decisions. Beyond the knowledge of correlations between mental illness and

84 gastrointestinal distress (which may already be used in many settings), predictive validity

85 of these associations may enable more efficient and accurate use of this knowledge for

86 decision-making in individual cases. In the present study, we work to establish the

87 generalizability and predictive validity of such associations prerequisite for their use in

88 serving youth mental health needs.

\section{Methods}

90 Participants

91 We analyzed data from three cohorts in this study (see Tables 1-3). Two of these

92 cohorts were collected by our lab in two cities: Los Angeles (LA, N = 327), and New 
93 York City (NYC, $\mathrm{N}=102)$. The third was a publicly available dataset based in New York

94 City: Healthy Brain Network (HBN, N = 1957; Alexander et al., 2017; Das et al., 2012).

95 Each cohort included children and adolescents between ages 3-21 years. In the LA and

96 NYC cohorts, some youth had experienced caregiving adversity (institutional or foster

97 care). In the HBN cohort, recruitment strategies (community-referred recruitment to

98 increase participation of families who were concerned about the psychiatric health of

99 their children) resulted in a higher proportion of participants meeting clinical diagnoses

100 for mental illness than expected in the general population.

101 Associations between gastrointestinal symptoms and anxiety were previously

102 published in the LA cohort (Callaghan et al., 2020). Here, we analyzed data from the LA

103 and NYC cohorts, before preregistering replication and predictive modeling analyses for

104 the HBN cohort (https://osf.io/687ky). We completed most preregistered analyses as

105 planned and acknowledge several differences in the supplement. Within the HBN dataset,

106 we analyzed both complete-case data (reported in analyses in the main manuscript) and

107 all data using imputation strategies (supplemental) in parallel. Before conducting

108 analyses, we split the HBN dataset into a training set (75\% of the participants) and a

109 holdout set ( $75 \%$ of the participants). Henceforth, we refer to 'HBN training data' to

110 mean the training set, 'HBN holdout data' to mean the holdout set, and 'HBN full

111 dataset' to mean all of the available HBN data (both training and holdout).

112

113 Child gastrointestinal symptoms

114 In all cohorts, we assessed child gastrointestinal (GI) symptomatology using

115 parent reports from the CBCL for ages 1.5-5 and 4-18 years (Achenbach, 1991). We 
116 included 4 items present in both versions so that symptoms could be compared across

117 ages. Using a 3 -point scale $(0=$ not true, $1=$ somewhat true, $2=$ very true $)$ to indicate the

118 degree to which their children were experiencing "physical problems without known

119 medical cause", parents reported incidence of nausea, stomachaches/cramps, vomiting,

120 and constipation (supplemental methods). The internal consistency of these four items, as

121 measured by Cronbach's alpha, was $0.63(95 \% \mathrm{CI}[0.61,0.66])$ in the full $\mathrm{HBN}$ cohort,

$1220.45(95 \%$ CI $[0.37,0.54])$ in the LA cohort, and $0.47(95 \%$ CI $[0.33,0.62]$ in the NYC

123 cohort. We used sum scores of these four items to calculate each participant's

124 gastrointestinal symptom score. While previous work (Callaghan et al., 2020) within the

125 LA cohort included one item from the Revised Child Anxiety and Depression Scale

126 (RCADS; Chorpita et al., 2000) within total GI scores, RCADS data were not available

127 for the HBN and NYC cohorts, so we only used CBCL items for the present study.

\section{Child anxiety symptoms}

129 Parent report: We measured anxiety-related behaviors in all cohorts using the

130 Screen for Child Anxiety Related Disorders-Parent version (SCARED-P; Birmaher et al.,

131 1997). Parents reported frequencies of each of 41 behaviors on this measure using a 3-

132 point scale $(0=$ never, $1=$ sometimes, $2=$ often $)$. We removed item \#11 ('my child gets

133 stomachaches at school') to avoid including explicit gastrointestinal symptoms in our

134 anxiety measure. Scores on this measure were summed (see Table 1). Cronbach's alpha

135 for the SCARED-P was 0.93 (95\% CI $[0.92,0.93])$ for the full HBN cohort, $0.92(95 \%$

136 CI $[0.91,0.94]$ for the LA Cohort, and $0.92(95 \%$ CI $[0.90,0.94]$ for the NYC cohort. 
137 Child report: In the HBN cohort only, youth reported on their own anxiety-

138 related behaviors using a child version of the SCARED scale (SCARED-C; Birmaher et

139 al., 1997). We summed anxiety symptom scores on SCARED-C using the same process

140 as with the parent-reported version. Cronbach's alpha for the SCARED-C was 0.94 (95\%

141 CI $[0.94,0.95)$.

142 Consensus clinical diagnoses: In the HBN cohort, youth completed a

143 computerized web-based version of the Kiddie Schedule for Affective Disorders and

144 Schizophrenia-Children's version (KSADS; Kaufman et al., 1997), and both youth and

145 parents completed semi-structured DSM-5-based psychiatric interviews with a licensed

146 clinician (KSADS-COMP). A clinical team reviewed these materials, as well as relevant

147 materials collected during study participation to arrive at a consensus for diagnoses of

148 DSM-5 disorders. We created a binary variable indicating if each participant was given

149 any one of 13 anxiety-related diagnoses (supplemental methods).

151 Replication \& Robustness

152 To replicate associations between GI symptoms and anxiety previously reported

153 (Callaghan et al., 2020), in each cohort (LA, NYC, and HBN training data) we regressed

154 continuous SCARED-P (parent-reported) scores on GI symptoms, sex, age, and

155 interactions of GI symptoms and sex using the rstanarm package in R (Goodrich et al.,

156 2018). To test robustness of such associations to different reporters of anxiety symptoms

157 in the HBN training data, we fit a similar linear regression model to continuous 
158 SCARED-C (child reported) scores, and a logistic regression to model whether

159 participants were diagnosed through clinician consensus using the KSADS.

160

161 Predictive Utility

162 Step 1: Model selection using the HBN training set: For each anxiety outcome

163 we selected best-performing models across several regression model formulations for

164 validation in our holdout sets (see Figure 1 and Step 2 below). The four model

165 formulations included (1) a summed total GI score plus age and sex regressors, (2) each

166 GI item as a separate regressor, plus age and sex regressors, (3) no GI terms with only

167 age and sex regressors, and (4) an intercept-only model (i.e. predicting the group average

168 level of anxiety for each person, included as a baseline comparison). For each

169 formulation including a GI term (1-2), models contained terms for age and sex, and we

170 also examined whether including interaction terms with age and sex (i.e. GI*age, GI*sex,

171 GI*age*sex) would improve model performance (see supplemental methods). We also

172 used models from formulation 2 to examine which GI items were most predictive of

173 anxiety (eFigures 18-22). From each of the four model formulations, we selected the

174 best-performing model from cross-validation in the training set, then fit best-performing

175 model variants to the entire training set (eTable 5) before validation in the holdout sets

176 (both the HBN holdout set and the combined LA + NYC data).

177 Step 2: Model Validation with holdout data: For each selected linear and

178 logistic regression model from step 1, we examined out-of-sample predictive

179 performance on the HBN holdout set as well as the combined LA and NYC cohorts (for 
180 SCARED-P). Performance was assessed in step 2 through bootstrapping and permutation

181 testing with $\mathrm{q}^{2}$ (proportion of variance predicted, see supplemental methods), Log Loss,

182 and Receiver-Operating Characteristic curves (ROC, and corresponding area under the

183 curve (AUC)) metrics. Because we made 6 comparisons with each set of models (all four

184 selected model formulations against the permuted null, plus both GI model formulations

185 against the No-GI model), we used a Bonferroni adjusted alpha level of $0.0083(.05 / 6)$ for 186 each permutation test. 




189 Figure 1: Flowchart summarizing our procedures and results for measuring the 190 predictive validity of models predicting anxiety from GI symptoms. Step 1: Model

191 Selection includes the models that were built on the HBN training dataset. There were 4 192 different model formulations built on the outcome measures: (1) GI Summed (including 193 age \& sex), (2) GI Individual Items (including age \& sex), (3) No GI (just age \& sex), (4)

194 Intercept only. Within those model formulations, several model variants were tested 195 which included models with no interactions between predictors, as well as models that 196 interacted with GI symptoms, age, sex, or included three-way interactions with GI 
197 symptoms age and sex. One model variant was selected for each model formulation and

198 carried through to Step 2: model validation. Step 2: Model validation occurred on the

199 holdout data (HBN test dataset, as well as the combined LA + NYC cohorts). The LA and

200 NYC cohorts were combined into a single holdout dataset due to highly similar

201 demographics, and to increase the sample size. Some of the models (i.e., those built on

202 the Clinical Cutoff scores from the SCARED-C and SCARED-P within the HBN training

203 data - see boxes surrounded by broken lines) were tested within the HBN holdout data,

204 but were not discussed in the main manuscript and are not represented in the colored

205 boxes to the bottom right of the graph. Colored boxes in the bottom right of the figure

206 represent the relative level of evidence for the predictive validity of each model for each

207 of the validation tests (>No-GI: better than a model with no GI symptoms included;

208 >Baseline: better than baseline threshold formulation, e.g., AUC $=0.5$; > Null model:

209 better than chance performance based on a permuted null distribution). The level of

210 evidence represented is relative, rather than absolute, meaning that relative to the other

211 models, those highlighted in green provide the strongest evidence for predictive

212 performance of GI models.

213

214 Exploratory Analyses: GI symptoms as a predictor for broader psychopathology

215 We also asked whether GI symptoms could function as a predictor of general

216 psychopathology risk within the HBN full dataset. We constructed logistic regression

217 models using summed GI symptoms as well as age and sex covariates to explore

218 associations between GI symptoms and the likelihood of any diagnosis, as well as each

219 respective KSADS DSM-5 diagnosis that occurred in more than 5\% of participants. In a

220 separate set of models with only GI sum term (and without age and sex predictors), we

221 examined predictive performance through repeated cross-validation (eFigure 30). 

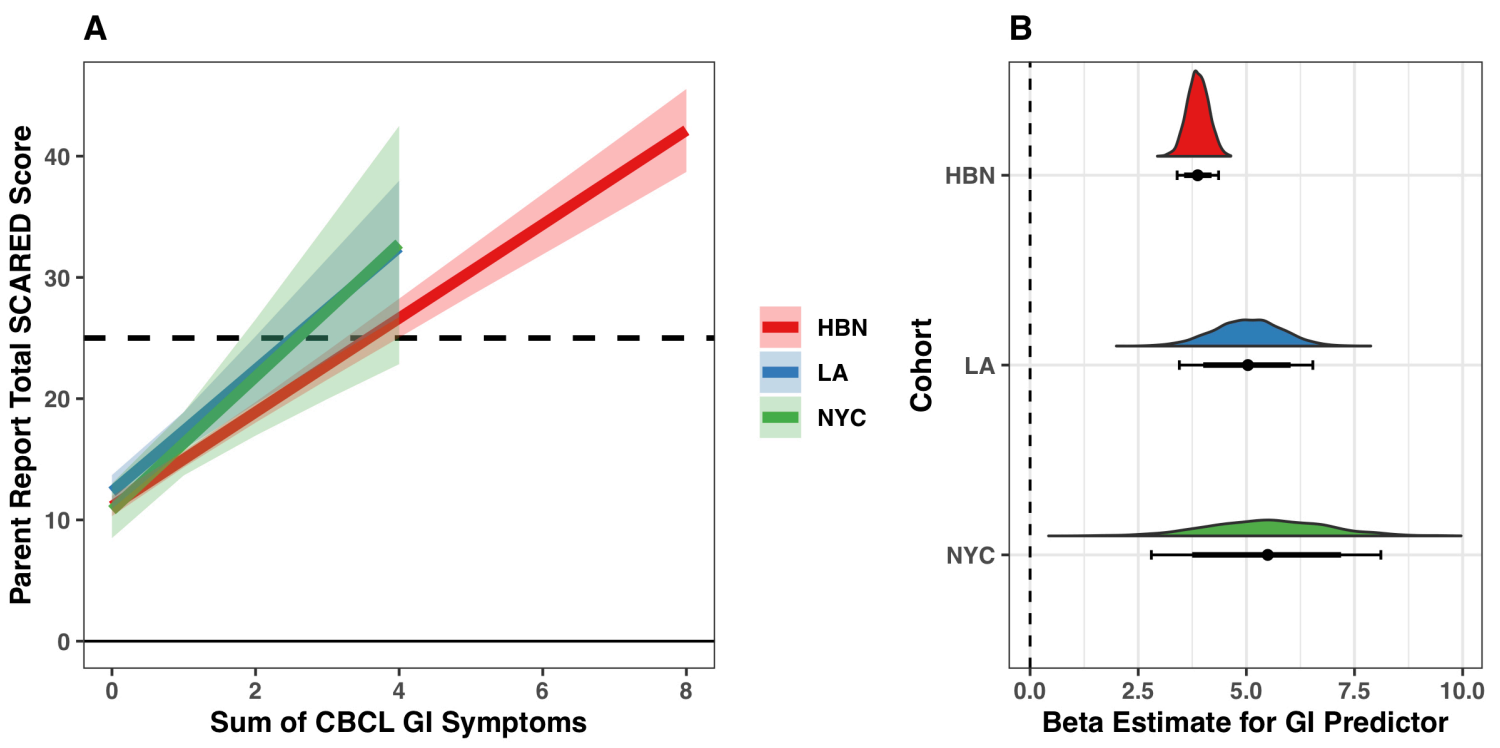

Figure 2: Replication of linear associations between GI symptoms and anxiety across the NYC (green) and LA (blue) cohorts, and HBN training data (red): A Fitted model predictions (solid lines) plus 95\% uncertainty intervals (semi-transparent overlays) for linear regressions fit to each of the three cohorts. Dotted line represents a score of 25, which is the clinical threshold for this measure. Predictions for NYC and LA cohorts are cut off above a GI sum score of 4 because no participants in these datasets scored over 4 for GI symptoms. Model predictions are displayed for an average participant of age 10.5 years. B: Posterior distributions for the GI sum score predictor in the linear regression models fit to each cohort (colored distributions). Underneath the colored distributions are the $80 \%$ (thick line) and $95 \%$ (thin line) uncertainty intervals. The posterior estimates for the GI predictor represent the predicted increase in SCARED-P scores associated with a 1 -unit increase in GI symptoms for an average participant of age 10.5 years. The dotted line at 0 indicates no estimated average change in SCARED-P scores as a function of GI symptoms.

228 average, higher total GI symptoms were associated with more parent-reported child

229 anxiety symptoms $\left(\hat{\beta}_{H B N}=3.88,95 \%\right.$ CI $[3.37,4.38], \hat{\beta}_{L A}=5.02,95 \%$ CI $[3.46,6.52]$,

231 addition, we found that associations between GI symptoms and anxiety were robust to

232 responder, such that within the HBN training dataset higher GI symptoms were on 
233 average associated with higher anxiety behaviors on SCARED-C $(\hat{\beta}=1.79,95 \%$ CI

$234[1.07,2.51])$, and higher probability of diagnosis on the $\operatorname{KSADS}(\hat{\beta}=0.28,95 \% \mathrm{CI}[0.19$,

$2350.37])$.
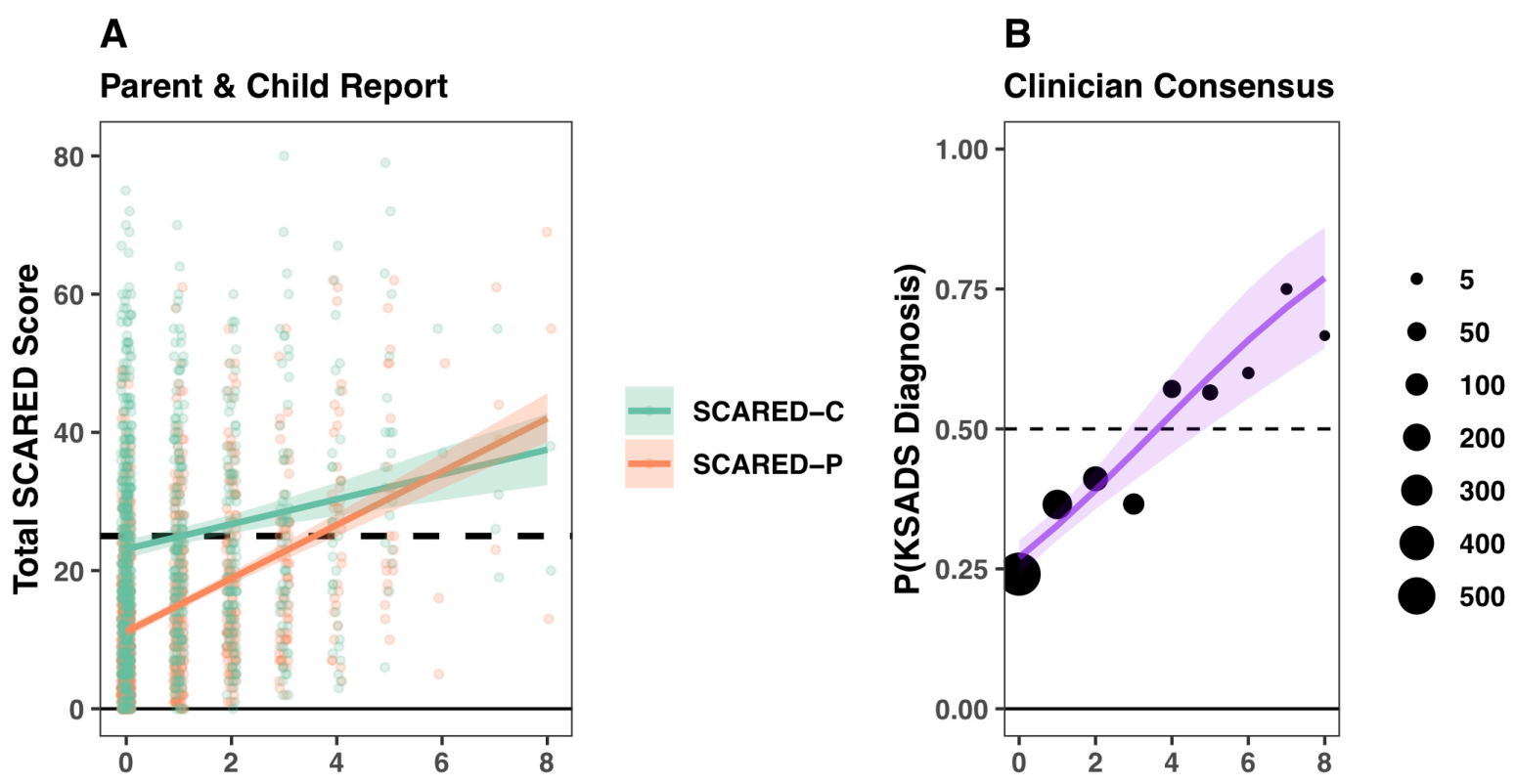

Figure 3: Robustness of associations between parent reports of child GI symptoms and both child-reported and clinician-evaluated anxiety in the HBN training set. All panels show model fitted predictions (solid lines) for an average participant of age 10.5 years with a $95 \%$ uncertainty interval (semi-transparent overlay). A: Linear regression with parent-reported anxiety behaviors on SCARED-P (orange) and child-reported anxiety behaviors on SCARED-C (green) as the outcomes. Points show individual participant scores on each measure. B: Logistic regression with clinician-consensus anxiety diagnosis based on the KSADS as the outcome. Points indicate proportions of participants at each possible GI score with diagnoses, and point size indicates the number of participants in a given bin. GI terms, inclusion of age/sex interaction terms had little impact on model performance. 
241 Through cross-validation with the HBN training data, we found that both the best-

242 performing GI summed and GI individual items models predicted anxiety scores on the

243 SCARED-P better than the best-performing No-GI model or intercept-only model (Figure

244 4). There were not consistent performance differences between the best GI summed

245 model and best GI individual items model. While GI models also tended to perform

246 better than No-GI models during cross-validation with the SCARED-C and KSADS

247 outcomes, they did not do so as consistently (eFigures 15-17).

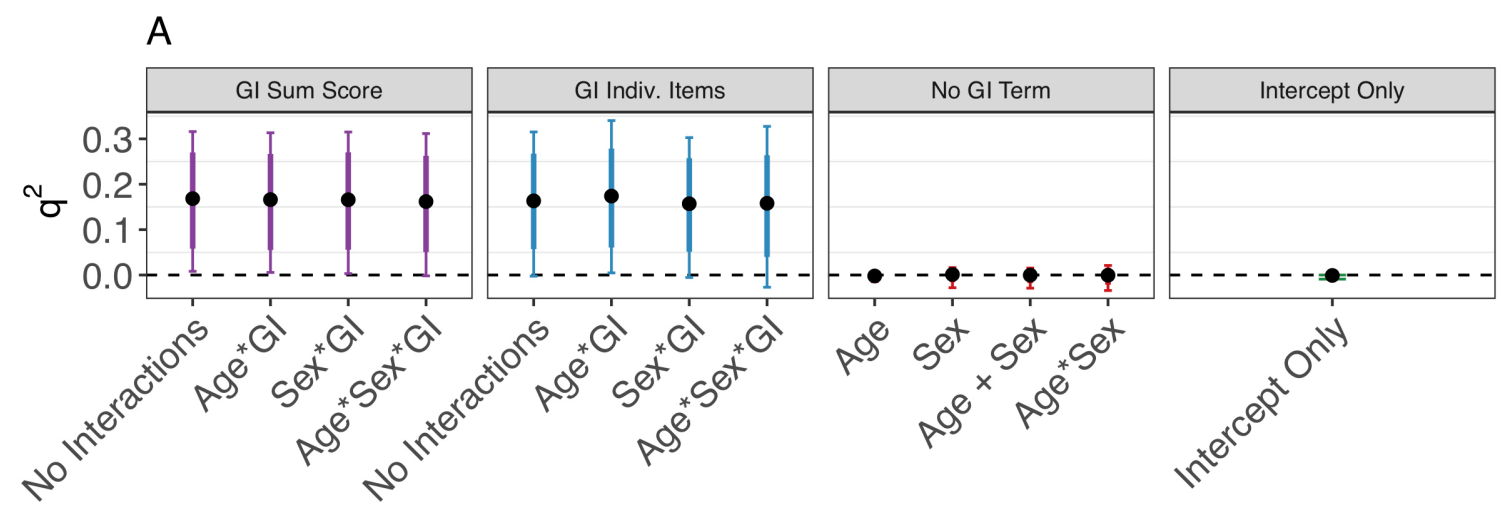

249
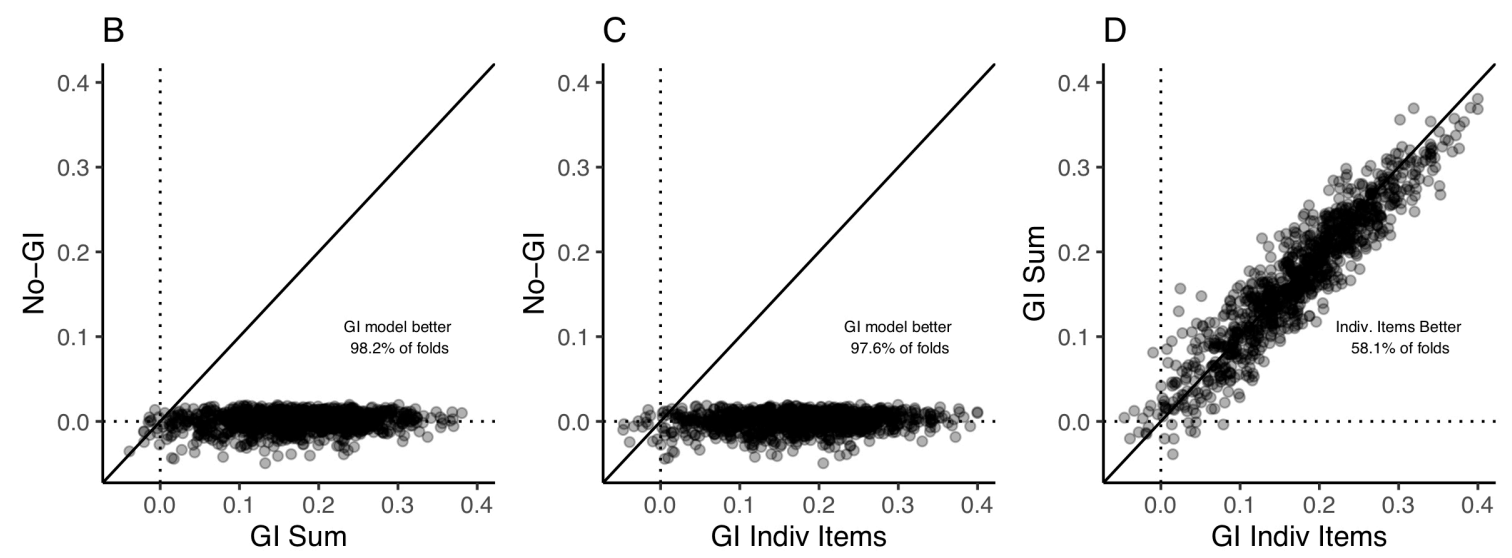

Figure 4: Cross-validation of linear regression models predicting SCARED-P from GI symptoms within the HBN training data. A: For each model formulation (GI summed, GI individual items, No GI Term, and Intercept Only, represented by the different boxes) the median $\mathrm{q}^{2}, 80 \%$ uncertainty interval (thick line), and $95 \%$ uncertainty interval (thin line) 
from all cross-validated folds are plotted for each model variant. B, C, and D: $\mathrm{q}^{2}$, calculated for the model variants selected in Step 1 across all folds (each data point represents a fold). Data points that fall above the line indicate a higher $\mathrm{q}^{2}$, for the model represented on the $y$-axis, whereas those that fall below the solid line indicate higher $\mathrm{q}^{2}$, from the model represented on the x-axis. Both the GI summed model (B) and the GI individual items model $(\mathbf{C})$ outperformed the No-GI model, and there were no consistent differences in performance between the two types of GI models (individual items and summed; D).

252 per the SCARED-P) in the HBN holdout data, GI summed $\left(q^{2}\right.$ median $=0.139,95 \% \mathrm{CI}$

$253[0.036,0.23])$ and GI individual items $\left(q^{2}\right.$ median $=0.144,95 \%$ CI $\left.[0.034,0.242]\right)$ models

254 both predicted variance over baseline, and outperformed the No-GI model $\left(q^{2}\right.$ median $=$

$2550.005,95 \%$ CI $[-0.011,0.018])$ in predicting variance in anxiety $\left(\mathrm{p}_{\text {summed }}=0.0045\right.$,

$256 p_{\text {indiv. items }}=0.0049$; Figure 5A). For child-reported anxiety, GI summed $\left(q^{2}\right.$ median $=0.073$,

$25795 \%$ CI $[0.005,0.135])$ and GI individual items $\left(q^{2}{ }_{\text {median }}=0.071,95 \%\right.$ CI $[0.004$,

$2580.138])$, but not No-GI model formulations $\left(q^{2}\right.$ median $=0.045,95 \%$ CI $\left.[-0.005,0.091]\right)$

259 reliably predicted variance in anxiety symptoms over a baseline $q^{2}$ of 0 , though neither

260 GI model formulation consistently outperformed the No-GI model $\left(\mathrm{p}_{\text {summed }}=0.0904\right.$,

$261 \quad$ pindiv. items $=0.1467)$.

262 To assess whether performance of any models was above chance, anxiety

263 outcomes in the HBN holdout dataset were shuffled 10000 times to calculate empirical

264 null distributions and compared to actual performance of each model. For parent-reported

265 anxiety, both the GI sum model and the GI individual item model performed better than

266 the null $\left(\mathrm{p}_{\text {summed }}=0.0001, \mathrm{p}_{\text {indiv. items }}=0.0001\right)$, whereas the no GI model did not $\left(\mathrm{p}_{\mathrm{no} \mathrm{GI}}=\right.$

2670.0351 , Figure 5A), suggesting that only models including a GI term could predict

268 parent-reported anxiety at above chance levels. For child-reported anxiety, the GI 
269 summed, GI individual items, and No-GI model performed better than the null $\left(\mathrm{p}_{\text {summed }}=\right.$

$\left.2700.0001, \mathrm{p}_{\text {indiv. items }}=0.0001, \mathrm{p}_{\mathrm{no}} \mathrm{GI}=0.0001\right)$, suggesting that models based on age and sex

271 alone, as well as those which also included GI symptoms, predicted variance in child-

272 reported anxiety symptoms at above chance levels.

273 In terms of classification of anxiety diagnoses within the HBN holdout dataset

274 (KSADS), the GI summed $\left(\mathrm{AUC}_{\text {median }}=0.634,95 \% \mathrm{CI}[0.577,0.69]\right)$, GI individual

275 items $\left(\mathrm{AUC}_{\text {median }}=0.637,95 \% \mathrm{CI}[0.579,0.693]\right)$, and No-GI $\left(\mathrm{AUC}_{\text {median }}=0.602,95 \%\right.$

276 CI [0.543, 0.659]) models consistently performed above a baseline AUC of 0.5 ,

277 displaying some sensitivity and specificity (Figure 5B). In addition, all three models

278 outperformed the null in predicting clinician-consensus diagnoses of anxiety under both

279 AUC $\left(\mathrm{p}_{\text {summed }}=0.0001, \mathrm{p}_{\text {indiv. items }}=0.0001, \mathrm{p}_{\mathrm{no} \mathrm{GI}}=0.0004\right)$ and log loss metrics $\left(\mathrm{p}_{\text {summed }}\right.$

$280=0.0001, p_{\text {indiv. items }}=0.0001, \mathrm{p}_{\mathrm{no} \mathrm{GI}}=0.0002$, Figure $\left.5 \mathrm{C}-\mathrm{D}\right)$. While classification models

281 with a GI term performed numerically better than the No-GI model on average, they did

282 not consistently outperform the No-GI model in either AUC $\left(\mathrm{p}_{\text {summed }}=0.0433, \mathrm{p}_{\text {indiv. items }}\right.$

$283=0.0425)$ or $\log \operatorname{loss}$ metrics $\left(\mathrm{p}_{\text {summed }}=0.011, \mathrm{p}_{\text {indiv. items }}=0.0298\right)$.

284 Model validation results for the combined LA/NYC holdout data were largely

285 similar to those with the HBN holdout data, such that GI model formulations predicted

286 variance in the SCARED-P continuous outcome over both the null and the No-GI model

287 (eFigure 24), while performance was variable for classification based on clinical cutoff

288 scores (eFigure 25). 

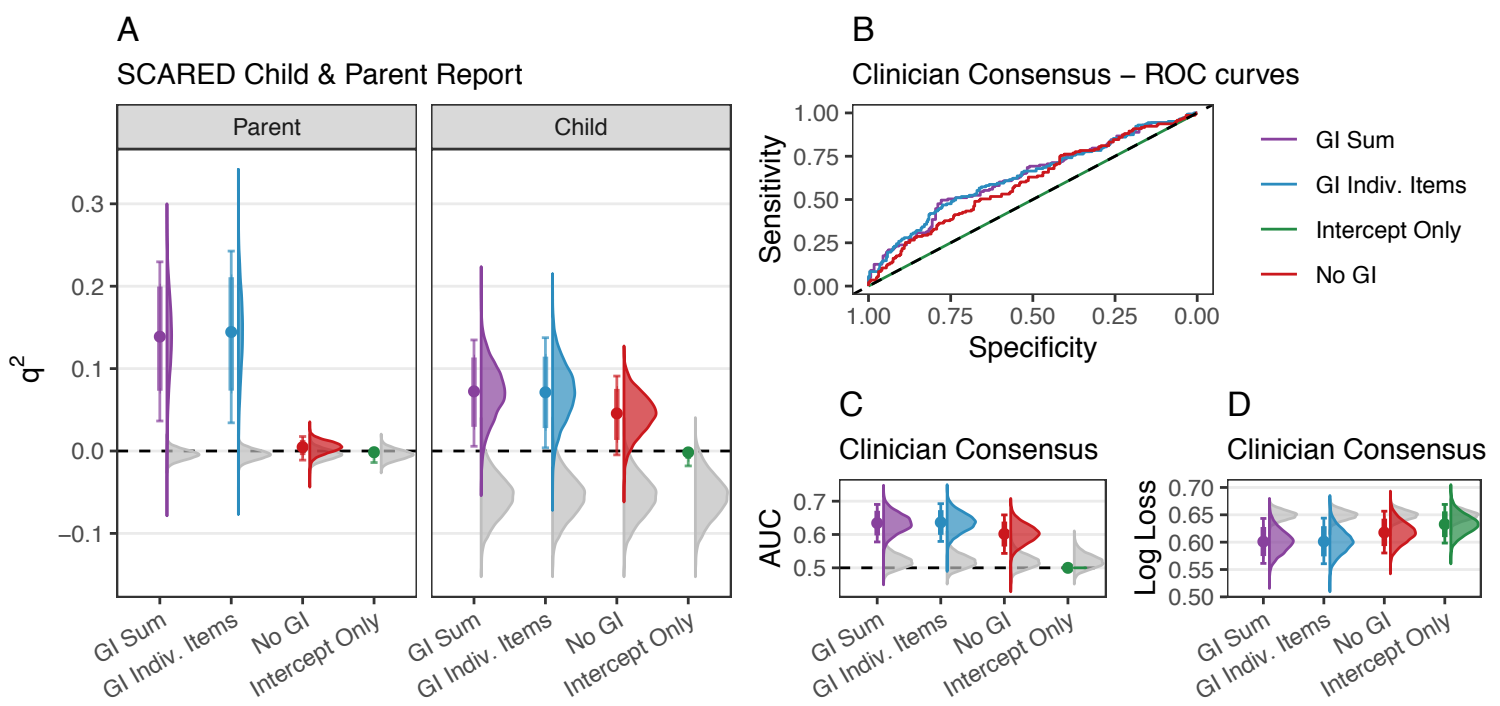

Figure 5: Bootstrapped model predictive performance and null permuted distributions on HBN holdout data for parent-report (SCARED-P, panel A), child-report (SCARED-C, panel A), and clinician consensus (KSADS) anxiety diagnoses (panel B-D). Bootstrapped distributions of model performance are shown in color (purple $=$ GI summed (model formulation 1), blue = GI individual items (model formulation 2), red = No-GI (model formulation 3), green = Intercept Only (model formulation 4)), and bootstrapped medians (colored points) are compared to the distribution of the best-performing null model (gray). A: Bootstrapped distributions of $\mathrm{q}^{2}$, metrics from resampling of the $\mathrm{HBN}$ holdout data for both parent-reported (SCARED-P) and child-reported (SCARED-C) anxiety symptoms. For the parent-reported anxiety, only GI models (GI summed and GI individual items) performed better than the null. For the child-reports, all models except for the intercept-only model tended to perform better than the null, with generally positive $\mathrm{q}^{2}$, scores. B: Receiver-operating characteristic curves for all model formulations for the full HBN holdout dataset. The $\mathrm{x}$-axis represents specificity (proportion of those true diagnoses correctly predicted), and the y-axis represents sensitivity (proportion of true diagnoses correctly predicted). The diagonal black line represents chance. $\mathbf{C}$ and $\mathbf{D}$ : Bootstrapped distributions of AUC (panel C) and log loss metrics (panel D) from resampling of the HBN holdout data for clinician-consensus diagnoses on the KSADS. GI summed, GI individual items, and No-GI models. 
While covarying for age and sex, GI symptoms were positively associated in the

294 full HBN dataset with several mental health diagnoses, notably in the categories of

295 anxiety, behavioral, elimination, and mood disorders (Figure 6A). Further, GI symptoms

296 were negatively associated with the likelihood of participants having no mental health

297 diagnoses. Additional cross-validation of logistic regression models with summed GI

298 symptoms as the sole predictor (no age or sex predictors) revealed evidence that several

299 diagnoses could be classified above a baseline AUC $=.5$ (Figure 6B).

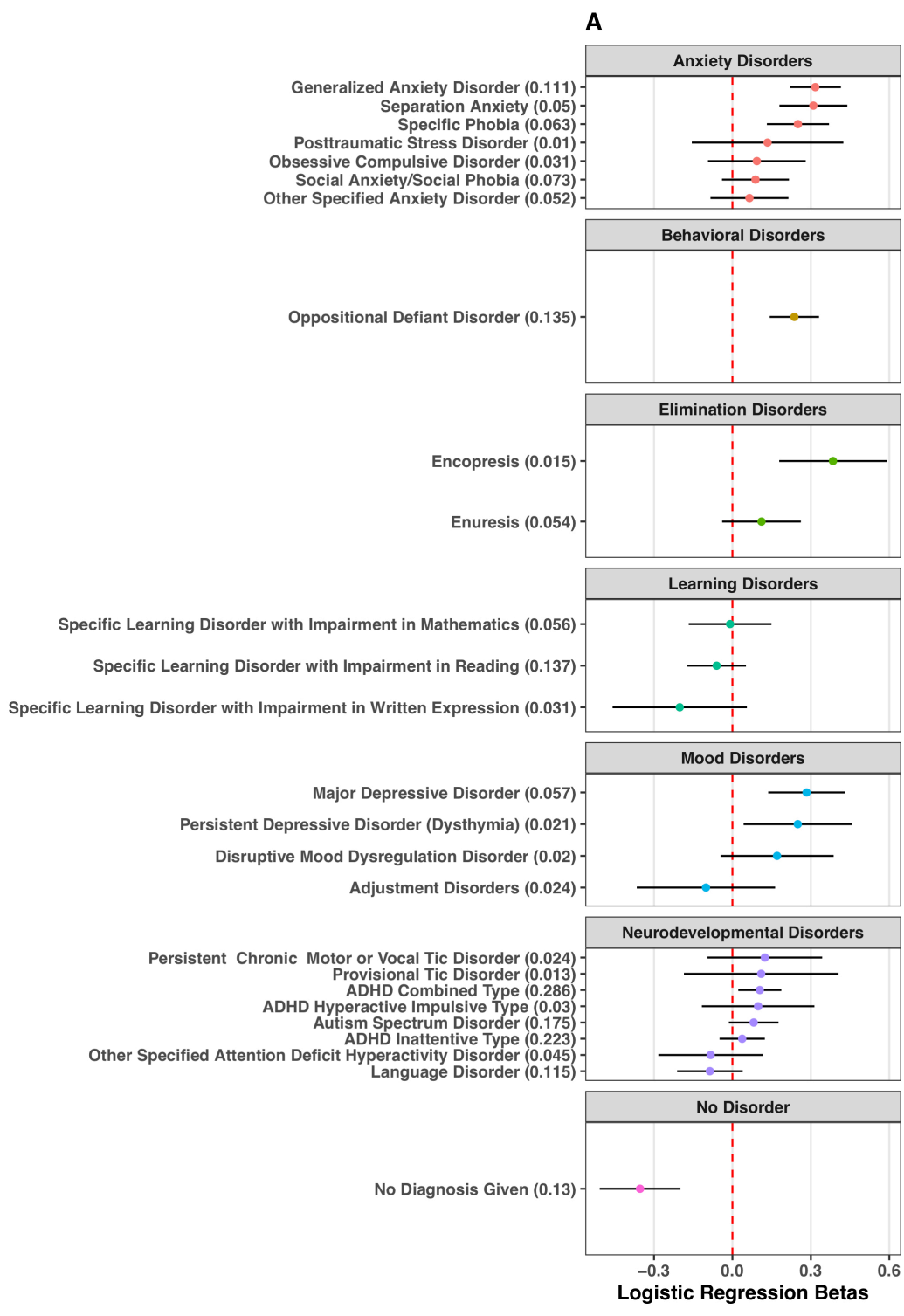

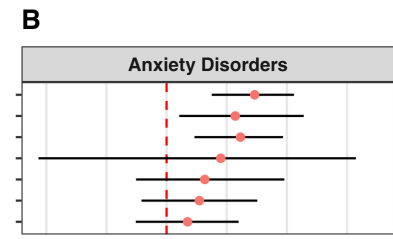

Behavioral Disorders
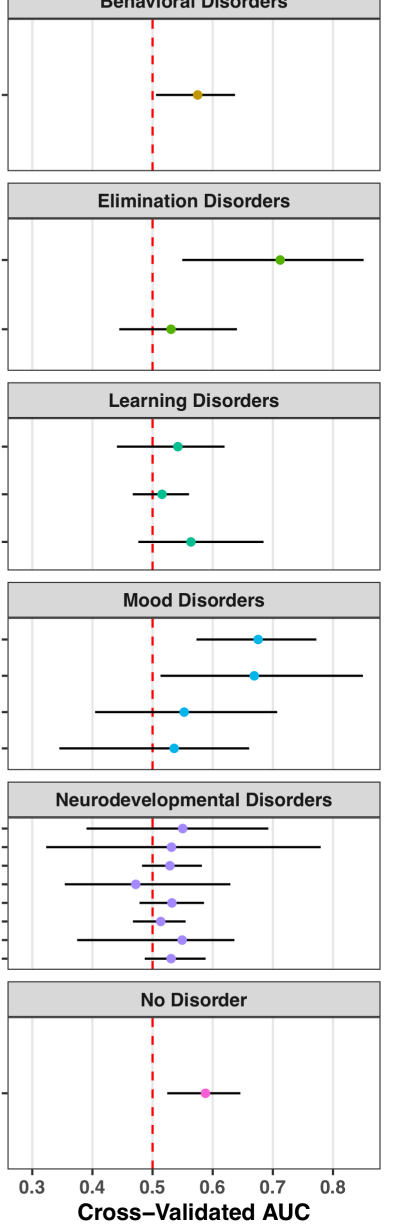
Figure 6: Exploring GI model performance for predicting a range of mental illness diagnoses (left y-axis). Numbers in parenthesis next to the name of each diagnosis (left $\mathrm{Y}$-axis) indicate the proportion of participants in the full HBN cohort with that diagnosis. Each outcome is grouped into a diagnostic category (box), with the grey title indicating the diagnostic category (e.g., 'behavioral disorders'). The beta estimate is indicated with a colored point and the lines around the point indicate the $95 \%$ uncertainty interval. A: Estimated logistic regression betas for a summed GI symptom term (including age \& sex covariate) in models fit to the entire HBN cohort, associated with likelihood of each respective outcome. Estimates are in log-odds. B: Cross-validated AUC estimates for each respective disorder from iterative rounds of cross-validation leaving out $25 \%$ of the HBN dataset. While the models in A included covariates for age and sex, cross-validated models only had a GI predictor, but no terms for age or sex to avoid inflated model performance based on age-related differences in specific psychopathologies. Points represent median AUC, and lines represent 95\% uncertainty intervals across rounds of cross-validation.

\section{Discussion}

Here we establish generalizability and predictive performance of GI-anxiety associations. We first attempted to replicate associations between gastrointestinal

304 symptoms and anxiety in several cohorts using different informants (parent, child,

305 clinician), to demonstrate the generalizability of these associations. We then assessed

306 predictive accuracy of gastrointestinal symptom models in holdout datasets. Across three

307 cohorts and with different informants, we found that gastrointestinal distress was

308 positively associated with youth anxiety, demonstrating a replicable and robust

309 relationship. These findings are consistent with prior work from other groups within both

310 adult and youth cohorts without enrichment for early life adversity (Haug et al., 2002;

311 Jones et al., 2017; Koloski et al., 2012; Waters et al., 2013). Models trained on one cohort

312 (HBN training set) were also successful in predicting anxiety outcomes above chance

313 levels in two holdout datasets (HBN holdout set and NYC+LA holdout set), although

314 predictive performance was quite variable. Specifically, in the HBN holdout data, models 
315 predicted anxiety at above chance levels for all anxiety informants. Further, GI models

316 outperformed equivalent models without gastrointestinal terms for parent-reported child

317 anxiety. However, models were less successful in predicting child-reported anxiety or

318 clinician-consensus diagnoses beyond what could already be predicted based on age and

319 sex. Similar findings emerged when the combined LA/NYC cohorts were used to test

320 model performance. Together, these findings establish both generalizability and

321 predictive validity of associations between gastrointestinal symptoms and anxiety.

322 While most of the present work focused on anxiety, exploratory analyses

323 indicated that GI symptoms were also associated with broader psychopathology within

324 the full HBN cohort. Models with a sole GI term predicted a range of clinician-evaluated

325 psychopathologies above a baseline $\mathrm{AUC}=0.5$, specifically generalized anxiety disorder,

326 separation anxiety, and specific phobias, major depressive disorder, dysthymia,

327 oppositional defiant disorder, and encopresis. While these findings are preliminary and

328 need to be confirmed in separate cohorts, links between GI symptoms and broader

329 psychopathology, mood disorders in particular, are consistent with prior work (Bagayogo

330 et al., 2013; Campo et al., 2004; Haug et al., 2002; Kennis et al., 2019; Okulate et al.,

331 2004). While such findings indicate that these models may lack specificity in their ability

332 to predict anxiety symptoms, they also suggest that somatic symptoms may be useful

333 tools for transdiagnostic referrals or treatment for child mental health (McGorry et al.,

334 2018; Weersing et al., 2012), which may in fact be preferred given the high comorbidity

335 in psychiatric presentations (Kessler et al., 1994). Indeed, comorbidity within the HBN

336 cohort was high. 
Given the associations between GI distress and anxiety, routine screening for

338 anxiety symptoms within all gastrointestinal distress presentations by pediatricians,

339 teachers, school nurses, and other child-facing professionals could likely help to increase

340 appropriate referrals (Cunningham et al., 2018). However, because these individuals at

341 initial access points for youth mental healthcare do not always have training, resources,

342 or time to administer such mental health evaluations, using GI symptoms to assess

343 psychopathology risk may be useful as a preliminary step. Particularly because somatic

344 symptoms may be readily observed by teachers and school nurses, and are often assessed

345 at primary care visits, increased attention to such symptoms as potential indicators of

346 mental health risk may help improve community monitoring of youth mental health.

347 Moreover, because anxiety symptom screening may miss youth who are exhibiting

348 prodromal or subthreshold anxiety, as well as youth who lack insight to report anxiety

349 symptoms, GI symptoms (either alone or in addition to anxiety screening) may help

350 identify youth who would benefit from psychiatric referral but may be missed by mental

351 distress screening.

352 Even with increasing awareness towards issues of mental health over time, many

353 key gatekeepers to community youth mental healthcare still express a lack of confidence

354 in their preparedness to identify youth psychopathology and their abilities to provide

355 adequate care (Green et al., 2019; Horwitz et al., 2015; Reinke et al., 2011). Based on the

356 generalizability and predictive validity of GI-anxiety associations investigated here, more

357 easily observable gastrointestinal complaints might then be one tool for addressing

358 difficulties in identifying children at risk for psychopathology, requiring little extra time

359 and training. Thus, while many calls have been issued for increasing mental health 
360 competencies in both pediatrics and in schools, increasing widespread intuitions about

361 relationships between physical and mental health symptoms serves as a relatively simple

362 and low-cost measure. Even small increases in knowledge of the interconnected nature of

363 mental and physical health may serve as an incremental addition to the skills and

364 confidence of child-facing professionals in identifying those at risk for psychopathology,

365 and improve community mental healthcare for youth (Green et al., 2019).

366 A key consideration for these data is the possibility that referring youth with

367 gastrointestinal distress to psychiatric services might impede an appropriate referral to

368 gastroenterology. Importantly, in this study, gastrointestinal complaints are presumed

369 non-organic (questions asked about symptoms with "no known medical cause").

370 Nevertheless, as psychological comorbidities in gastrointestinal presentations are

371 common in both organic and non-organic abdominal pain (Raymer et al., 1984), we

372 suggest that psychiatric referrals may remain appropriate for even organic abdominal

373 pain in youth and encourage concurrent screening for organic causes for gastrointestinal

374 pain.

375 Limitations

376 While the current work demonstrates the prerequisite generalizability of GI-

377 anxiety associations for future development of screening instruments, we regard such

378 models in their current form as valuable predictors of risk, but not stand-alone diagnostic

379 screening tools. Though our models often performed above chance levels in predicting

380 out-of-sample anxiety symptoms, such performance does not yet meet a level sufficient

381 for clinical utility as a diagnostic screener. Our GI model AUC values in the range of 0.6- 
3820.7 for classifying diagnoses and $10-15 \%$ predicted variance for continuous symptoms at

383 best, while reliably above chance, fall below standards for diagnostic screening

384 algorithms (Dobrow et al., 2018). In fact, despite that GI-anxiety associations have been

385 previously reported using similar measures within the LA cohort (Callaghan et al., 2020),

386 GI model prediction of whether youth met clinical threshold on the SCARED-P was not

387 reliably higher than chance in a holdout set of the combined LA + NYC cohorts (eFigure

388 24). Nevertheless, that GI models demonstrate predictive ability above chance provides

389 evidence for potential future uses of such approaches, particularly if more targeted GI

390 symptom questions are used, or in combination with other potentially informative

391 variables (such as other somatic distresses, sleep, early-life history).

A second limitation is that while GI models for parent-reported anxiety reliably

393 outperformed models based on age and sex alone (No-GI models), this was not

394 consistently true for child-reported anxiety or clinician-consensus diagnoses. One

395 explanation for this is that because gastrointestinal items were also parent-reported, better

396 predictive performance for parent-reported anxiety outcomes may have been driven by

397 parental reporting effects, rather than youth anxiety symptoms themselves. On the other

398 hand, stronger relative performance for GI models for the parent-reported anxiety

399 outcome could be attributable to the fact that parent-reported anxiety scores (SCARED-

400 P) were not strongly associated with age, while child-reported anxiety tended to decrease

401 with age, and clinician-consensus diagnoses tended to increase with age (see eTable 3 \&

402 eTable 4). Accordingly, models based on age and sex alone performed somewhat better

403 than the null for child-reported and clinician-diagnosed anxiety (see Figure 5). Consistent

404 with prior work (Carter, 2015; Carter et al., 2011; Crystal et al., 1994), females also had 
405 higher average anxiety symptoms in all three cohorts, and likelihood of anxiety diagnoses

406 in the HBN training set (see eFigure 4). Thus, despite the fact that including interaction

407 terms between GI symptoms and age or sex did not meaningfully improve model

408 performance during cross-validation within the HBN training data (see Figure 3 \&

409 eFigures 15-16), age and sex helped prediction of both child-reported and clinician-

410 diagnosed anxiety outcomes.

411 Relatedly, parent-reported gastrointestinal items were taken from a screening

412 measure for psychosocial development (CBCL) rather than from an instrument designed

413 to assess gastrointestinal distress (e.g., ROME IV). Because for each GI symptom,

414 response options were limited to 'not true', 'somewhat true', or 'very true', this measure

415 lacks the precision to measure nuanced differences in gastrointestinal distress. Even when

416 we summed all four of these GI items to create a scale from $0-8$, scores above 3 were rare

417 in all cohorts, and no participants scored above 4 in the LA or NYC cohorts (see

418 truncated model fits in Figure 2). In addition, this 4-item composite demonstrated poor

419 internal consistency, which may have hampered its use in predictive models. Better

420 performance might be expected in future models where predictors are designed to capture

421 more specific gastrointestinal distress. Future work could also leverage child-reported GI

422 symptoms and/or biological measures associated with GI distress (e.g., the gut

423 microbiota) to mitigate potential biases in parent reporting of child gastrointestinal

424 distress and improve prediction of youth anxiety.

425 Additionally, in the current study we evaluate the ability of models to predict

426 concurrent anxiety as an outcome, rather than future anxiety symptoms. While we believe

427 there is value in this approach for informing community mental healthcare, work is also 
428 needed to evaluate model predictive performance for future anxiety in longitudinal 429 datasets.

430 Another limitation is that models performed best in predictions of continuous

431 anxiety symptoms in both holdout sets, rather than classification of binary anxiety

432 diagnoses. While such performance does fit with spectrum-based conceptualizations of

433 mental illness (i.e. Research Domain Criteria), future iterations of models that employ

434 more specific assessments of gastrointestinal distress should continue to monitor

435 predictive performance on continuous and discrete outcomes.

436 A final concern regards the generalizability of the cohorts assessed here. The LA

437 and NYC cohorts included youth who had experienced institutional or foster care abroad,

438 both of which have been associated with gastrointestinal distress and elevated anxiety

439 (Bos et al., 2011; Bradford et al., 2012). The HBN cohort employed sampling strategies

440 that lead to elevated rates of psychopathology relative to the population. In all cohorts,

441 distributions of annual family income were well above local (NYC or LA) medians (see

442 Table 1 \& eFigure 2), and there were more White participants than any other racial group 443 (see Table 2).

444 An important consideration in relation to the racial and ethnic compositions of the 445 samples included here is that prior work has shown that somatic symptom expression in 446 youth can vary by ethnicity. For example, Latino youth of some ethnocultural

447 backgrounds express more somatic anxiety symptoms relative to European-American

448 youth (Pina \& Silverman, 2004; Varela et al., 2008; Varela \& Hensley-Maloney, 2009).

449 In our study, we did not find consistent evidence of differences in associations between

450 GI symptoms and anxiety (see eFigures 13-14) or model performance (see eFigures 26- 
45129 ) as a function of ethnicity or race. However, the absence of such differences may have

452 been a consequence of the relatively low numbers of non-White, and Hispanic or Latino

453 youth (see Tables 1-2), or because the racial and ethnic categorizations analyzed may not

454 have been sufficiently nuanced to detect differences in youth anxiety expression across

455 sociocultural communities (Alegria et al., 2019; Quinones-Camacho, 2018). As with

456 other clinical measures, development of tools assessing youth anxiety risk must consider

457 differences in symptom expression across cultures, as well as how to prevent interactions

458 with existing systems of systemic discrimination that perpetuate further inequity in

459 referral and treatment (Chen et al., 2019; Merikangas et al., 2011; Obermeyer et al., 2019;

$460 \mathrm{Yu} \&$ Kohane, 2019). To this end, future studies will need to test model performance in

461 cohorts with representation of mental illness, caregiving experience, race, and ethnicity

462 background that more closely parallels the populations seen in community settings.

\section{Code and data availability}

465 Code used for all data preprocessing and analyses is available at

466 https://github.com/pab2163/giAnxiety. Further information on Data Usage Agreements

467 for Healthy Brain Network data can be found at

468 http://fcon_1000.projects.nitrc.org/indi/cmi_healthy_brain_network/Pheno_Access.html.

\section{Citation Diversity Statement}


474 number of such papers in the field (Ambekar et al., 2009; Caplar et al., 2017; Dion et al.,

475 2018; Dworkin et al., 2020; Maliniak et al., 2013). Here we sought to proactively

476 consider choosing references that reflect the diversity of the field in thought, form of

477 contribution, gender, race, ethnicity, and other factors. First, we obtained the predicted

478 gender of the first and last author of each reference by using databases that store the

479 probability of a first name being carried by a woman (Dworkin et al., 2020; Zhou et al.,

480 2020). By this measure (and excluding self-citations to the first and last authors of our

481 current paper), our references contain $33.63 \%$ woman(first)/woman(last), $17.55 \%$

$482 \mathrm{man} /$ woman, $24.78 \%$ woman/man, and 24.04\% man $/ \mathrm{man}$. This method is limited in that

483 a) names, pronouns, and social media profiles used to construct the databases may not, in

484 every case, be indicative of gender identity and b) it cannot account for intersex, non-

485 binary, or transgender people. Second, we obtained predicted racial/ethnic category of the

486 first and last author of each reference by databases that store the probability of a first and

487 last name being carried by an author of color (Ambekar et al., 2009; Sood \&

488 Laohaprapanon, 2018). By this measure (and excluding self-citations), our references

489 contain $11.54 \%$ author of color (first)/author of color(last), 13.63\% white author/author

490 of color, $25.01 \%$ author of color/white author, and $49.83 \%$ white author/white author.

491 This method is limited in that a) names and Florida Voter Data to make the predictions

492 may not be indicative of racial/ethnic identity, and b) it cannot account for Indigenous

493 and mixed-race authors, or those who may face differential biases due to the ambiguous

494 racialization or ethnicization of their names. We look forward to future work that could

495 help us to better understand how to support equitable practices in science. 
Achenbach, T. M. (1991). Integrative Guide for the 1991 CBCL/4-18, Ysr, and Trf Profiles (1st US-1st Printing edition). Univ Vermont/Dept Psychiatry.

Achenbach, T. M., \& Edelbrock, C. S. (1983). Manual for the child behavior checklist and revised child behavior profile. T.M. Achenbach.

Alegria, M., Shrout, P. E., Canino, G., Alvarez, K., Wang, Y., Bird, H., Markle, S. L., Ramos-Olazagasti, M., Rivera, D. V., Cook, B. L., Musa, G. J., Falgas-Bague, I., NeMoyer, A., Dominique, G., \& Duarte, C. (2019). The effect of minority status and social context on the development of depression and anxiety: A longitudinal study of Puerto Rican descent youth. World Psychiatry, 18(3), 298-307.

Alexander, L. M., Escalera, J., Ai, L., Andreotti, C., Febre, K., Mangone, A., VegaPotler, N., Langer, N., Alexander, A., Kovacs, M., Litke, S., O’Hagan, B.,

514 Ambekar, A., Ward, C., Mohammed, J., Male, S., \& Skiena, S. (2009). Name-ethnicity Andersen, J., Bronstein, B., Bui, A., Bushey, M., Butler, H., Castagna, V., classification from open sources. Proceedings of the 15th ACM SIGKDD International Conference on Knowledge Discovery and Data Mining, 49-58. https://doi.org/10.1145/1557019.1557032 
518 Bagayogo, I. P., Interian, A., \& Escobar, J. I. (2013). Transcultural Aspects of Somatic 519 Symptoms in the Context of Depressive Disorders. Cultural Psychiatry, 33, 64520 74. https://doi.org/10.1159/000350057

521 Beesdo-Baum, K., \& Knappe, S. (2012). Developmental Epidemiology of Anxiety 522 Disorders. Child and Adolescent Psychiatric Clinics, 21(3), 457-478.

$523 \quad$ https://doi.org/10.1016/j.chc.2012.05.001

524 Bell, C. C., \& McKay, M. M. (2004). Constructing a Children's Mental Health Infrastructure Using Community Psychiatry Principles. Journal of Legal Medicine, 25(1), 5-22. https://doi.org/10.1080/01947640490361808

527 Birmaher, B., Khetarpal, S., Brent, D., Cully, M., Balach, L., Kaufman, J., \& Neer, S. M.

528 (1997). The Screen for Child Anxiety Related Emotional Disorders (SCARED): Scale Construction and Psychometric Characteristics. Journal of the American Academy of Child \& Adolescent Psychiatry, 36(4), 545-553. https://doi.org/10.1097/00004583-199704000-00018

532 Bodas, J., Ollendick, T. H., \& Sovani, A. V. (2008). Test anxiety in Indian children: A 533 cross-cultural perspective. Anxiety, Stress, \& Coping, 21(4), 387-404.

535 Bos, K., Zeanah, C. H., Fox, N. A., Drury, S. S., McLaughlin, K. A., \& Nelson, C. A. (2011). Psychiatric Outcomes in Young Children with a History of Institutionalization. Harvard Review of Psychiatry, 19(1), 15-24. https://doi.org/10.3109/10673229.2011.549773

539 Bradford, K., Shih, W., Videlock, E. J., Presson, A. P., Naliboff, B. D., Mayer, E. A., \& 540 Chang, L. (2012). Association Between Early Adverse Life Events and Irritable 
Bowel Syndrome. Clinical Gastroenterology and Hepatology, 10(4), 385-390.e3. https://doi.org/10.1016/j.cgh.2011.12.018

543 Callaghan, B. L., Fields, A., Gee, D. G., Gabard-Durnam, L., Caldera, C., Humphreys, K.

544 L., Goff, B., Flannery, J., Telzer, E. H., Shapiro, M., \& Tottenham, N. (2020). Mind and gut: Associations between mood and gastrointestinal distress in children exposed to adversity. Development and Psychopathology, 32(1), 309-328.

548 Callaghan, B. L., Fields, A., Gee, D. G., Gabard-Durnam, L., Caldera, C., Humphreys, K. 549 L., Goff, B., Flannery, J., Telzer, E. H., Shapiro, M., \& Tottenham, N. https://doi.org/10.1017/S0954579419000087

553 Campo, J. V., Bridge, J., Ehmann, M., Altman, S., Lucas, A., Birmaher, B., Lorenzo, C. 554 D., Iyengar, S., \& Brent, D. A. (2004). Recurrent Abdominal Pain, Anxiety, and 555 Depression in Primary Care. Pediatrics, 113(4), 817-824.

556 https://doi.org/10.1542/peds.113.4.817

557 Caplar, N., Tacchella, S., \& Birrer, S. (2017). Quantitative evaluation of gender bias in 558 astronomical publications from citation counts. Nature Astronomy, 1(6), 1-5. 559 https://doi.org/10.1038/s41550-017-0141

560 Carter, R. (2015). Anxiety Symptoms in African American Youth: The Role of Puberty 561 and Biological Sex. The Journal of Early Adolescence, 35(3), 281-307.

562 https://doi.org/10.1177/0272431614530809 
563 Carter, R., Silverman, W. K., \& Jaccard, J. (2011). Sex Variations in Youth Anxiety

564 Symptoms: Effects of Pubertal Development and Gender Role Orientation.

565 Journal of Clinical Child \& Adolescent Psychology, 40(5), 730-741.

$566 \quad$ https://doi.org/10.1080/15374416.2011.597082

567 Chen, I. Y., Szolovits, P., \& Ghassemi, M. (2019). Can AI Help Reduce Disparities in

568 General Medical and Mental Health Care? AMA Journal of Ethics, 21(2), 167-

569 179. https://doi.org/10.1001/amajethics.2019.167

570 Chorpita, B. F., Yim, L., Moffitt, C., Umemoto, L. A., \& Francis, S. E. (2000).

571 Assessment of Symptoms of DSM-IV Anxiety and Depression in Children: A

572 Revised Child Anxiety and Depression Scale. Behaviour Research and Therapy,

$573 \quad 38(8), 835-855$. https://doi.org/10.1016/S0005-7967(99)00130-8

574 Cohen, P., Pine, D. S., Must, A., Kasen, S., \& Brook, J. (1998). Prospective Associations

575 between Somatic Illness and Mental Illness from Childhood to Adulthood.

$576 \quad$ American Journal of Epidemiology, 147(3), 232-239.

577 https://doi.org/10.1093/oxfordjournals.aje.a009442

578 Cooper, S., Valleley, R. J., Polaha, J., Begeny, J., \& Evans, J. H. (2006). Running Out of

579 Time: Physician Management of Behavioral Health Concerns in Rural Pediatric

580 Primary Care. Pediatrics, 118(1), e132-e138. https://doi.org/10.1542/peds.2005-

$581 \quad 2612$

582 Crystal, D. S., Chen, C., Fuligni, A. J., Stevenson, H. W., Hsu, C.-C., Ko, H.-J.,

583 Kitamura, S., \& Kimura, S. (1994). Psychological Maladjustment and Academic

584 Achievement: A Cross-Cultural Study of Japanese, Chinese, and American High 
School Students. Child Development, 65(3), 738-753. https://doi.org/10.1111/j.1467-8624.1994.tb00780.x

587 Cunningham, N. R., Moorman, E., Brown, C. M., Mallon, D., Chundi, P. K., Mara, C. A., 588 Pentiuk, S., Lynch-Jordan, A. M., Dykes, D. M. H., Elfers, J., \& Farrell, M. K. (2018). Integrating Psychological Screening Into Medical Care for Youth With Abdominal Pain. Pediatrics, 142(2). https://doi.org/10.1542/peds.2017-2876

Das, S., Zijdenbos, A. P., Harlap, J., Vins, D., \& Evans, A. C. (2012). LORIS: A WebBased Data Management System for Multi-Center Studies. Frontiers in Neuroinformatics, 5. https://doi.org/10.3389/fninf.2011.00037

594 Dion, M. L., Sumner, J. L., \& Mitchell, S. M. (2018). Gendered Citation Patterns across Political Science and Social Science Methodology Fields. Political Analysis, 26(3), 312-327. https://doi.org/10.1017/pan.2018.12

Dobrow, M. J., Hagens, V., Chafe, R., Sullivan, T., \& Rabeneck, L. (2018). Consolidated principles for screening based on a systematic review and consensus process. CMAJ: Canadian Medical Association Journal = Journal de l'Association Medicale Canadienne, 190(14), E422-E429. https://doi.org/10.1503/cmaj.171154

Dowdy, E., Furlong, M., Raines, T. C., Bovery, B., Kauffman, B., Kamphaus, R. W., Dever, B. V., Price, M., \& Murdock, J. (2015). Enhancing School-Based Mental Health Services With a Preventive and Promotive Approach to Universal Screening for Complete Mental Health. Journal of Educational and Psychological Consultation, 25(2-3), 178-197. https://doi.org/10.1080/10474412.2014.929951

Duong, M. T., Bruns, E. J., Lee, K., Cox, S., Coifman, J., Mayworm, A., \& Lyon, A. R. (2020). Rates of Mental Health Service Utilization by Children and Adolescents 
in Schools and Other Common Service Settings: A Systematic Review and MetaAnalysis. Administration and Policy in Mental Health and Mental Health Services Research. https://doi.org/10.1007/s10488-020-01080-9

611 Dvorsky, M. R., Girio-Herrera, E., \& Owens, J. S. (2014). School-Based Screening for Mental Health in Early Childhood. In M. D. Weist, N. A. Lever, C. P. Bradshaw, \& J. S. Owens (Eds.), Handbook of School Mental Health: Research, Training, Practice, and Policy (pp. 297-310). Springer US. https://doi.org/10.1007/978-1-

Dworkin, J. D., Linn, K. A., Teich, E. G., Zurn, P., Shinohara, R. T., \& Bassett, D. S. (2020). The extent and drivers of gender imbalance in neuroscience reference lists. Nature Neuroscience, 23(8), 918-926. https://doi.org/10.1038/s41593-0200658-y

Gabry, J., Ali, I., Brilleman, S., Novik, J. B., AstraZeneca, University, T. of C., Team (R/stan_aov.R), R. C. D., Bates (R/pp_data.R), D., Maechler (R/pp_data.R), M., Bolker (R/pp_data.R), B., Walker (R/pp_data.R), S., Ripley (R/stan_aov.R, B., R/stan_polr.R), Venables (R/stan_polr.R), W., Burkner (R/misc.R), P.-C., \& Goodrich, B. (2019). rstanarm: Bayesian Applied Regression Modeling via Stan (2.19.2) [Computer software]. https://CRAN.R-project.org/package=rstanarm Beers, L. S., \& Long, M. (2019). Increasing Mental Health Engagement From Primary Care: The Potential Role of Family Navigation. Pediatrics, 143(4). https://doi.org/10.1542/peds.2018-2418 
630 Green, C. M., Foy, J. M., Earls, M. F., \& Committee on Psychosocial Aspects of Child and Family Health, M. H. L. W. G. (2019). Achieving the Pediatric Mental Health Competencies. Pediatrics, 144(5). https://doi.org/10.1542/peds.2019-2758

633 Gudiño, O. G., Martinez, J. I., \& Lau, A. S. (2012). Mental Health Service Use by Youths in Contact With Child Welfare: Racial Disparities by Problem Type. Psychiatric Services, 63(10), 1004-1010. https://doi.org/10.1176/appi.ps.201100427

Haug, T. T., Mykletun, A., \& Dahl, A. A. (2002). Are Anxiety and Depression Related to Gastrointestinal Symptoms in the General Population? Scandinavian Journal of Gastroenterology, 37(3), 294-298. https://doi.org/10.1080/003655202317284192

Heneghan, A., Garner, A. S., Storfer-Isser, A., Kortepeter, K., Stein, R. E. K., \& Horwitz,

644 Horwitz, S. M., Storfer-Isser, A., Kerker, B. D., Szilagyi, M., Garner, A., O’Connor, K. and Adolescents: Do Pediatricians and Child and Adolescent Psychiatrists Agree? Functional Gastrointestinal Disorders in Patients but Not in the Population. Clinical Gastroenterology and Hepatology, 15(7), 1014-1020.e4. https://doi.org/10.1016/j.cgh.2016.12.032 
653 Kaufman, J., Birmaher, B., Brent, D., Rao, U., Flynn, C., Moreci, P., Williamson, D., \&

654 Ryan, N. (1997). Schedule for Affective Disorders and Schizophrenia for School-

655 Age Children-Present and Lifetime Version (K-SADS-PL): Initial reliability and

656 validity data. Journal of the American Academy of Child and Adolescent

$657 \quad$ Psychiatry, 36(7), 980-988. https://doi.org/10.1097/00004583-199707000-00021

658 Kennis, M., Gerritsen, L., van Dalen, M., Williams, A., Cuijpers, P., \& Bockting, C.

659 (2019). Prospective Biomarkers of Major Depressive Disorder: A Systematic

660 Review and Meta-Analysis. Molecular Psychiatry, 1-18.

$661 \quad$ https://doi.org/10.1038/s41380-019-0585-z

662 Kessler, R. C., Berglund, P., Demler, O., Jin, R., Merikangas, K. R., \& Walters, E. E.

663 (2005). Lifetime Prevalence and Age-of-Onset Distributions of DSM-IV

664 Disorders in the National Comorbidity Survey Replication. Archives of General

$665 \quad$ Psychiatry, 62(6), 593-602. https://doi.org/10.1001/archpsyc.62.6.593

666 Kessler, R. C., McGonagle, K. A., Zhao, S., Nelson, C. B., Hughes, M., Eshleman, S.,

667 Wittchen, H.-U., \& Kendler, K. S. (1994). Lifetime and 12-Month Prevalence of

668 DSM-III-R Psychiatric Disorders in the United States: Results From the National

669 Comorbidity Survey. Archives of General Psychiatry, 51(1), 8-19.

670 https://doi.org/10.1001/archpsyc.1994.03950010008002

671 Kim, J. H. J., Tsai, W., Kodish, T., Trung, L. T., Lau, A. S., \& Weiss, B. (2019). Cultural

672 variation in temporal associations among somatic complaints, anxiety, and

673 depressive symptoms in adolescence. Journal of Psychosomatic Research, 124,

674 109763. https://doi.org/10.1016/j.jpsychores.2019.109763 
675 Koloski, N. A., Jones, M., Kalantar, J., Weltman, M., Zaguirre, J., \& Talley, N. J. (2012).

676 The brain-gut pathway in functional gastrointestinal disorders is bidirectional: A

677 12-year prospective population-based study. Gut, 61(9), 1284-1290.

678 https://doi.org/10.1136/gutjnl-2011-300474

679 Loyd, A. B., Hotton, A. L., Walden, A. L., Kendall, A. D., Emerson, E., \& Donenberg,

680 G. R. (2019). Associations of ethnic/racial discrimination with internalizing

681 symptoms and externalizing behaviors among juvenile justice-involved youth of

682 color. Journal of Adolescence, 75, 138-150.

683 https://doi.org/10.1016/j.adolescence.2019.07.012

684 Luthar, S. S., Ebbert, A. M., \& Kumar, N. L. (2020). The Well-Being Index (WBI) for

685 schools: A brief measure of adolescents' mental health. Psychological

686 Assessment, 32(10), 903-914. https://doi.org/10.1037/pas0000913

687 Luthar, S. S., Kumar, N. L., \& Zillmer, N. (2020). Teachers' responsibilities for students'

688 mental health:Challenges in high achieving schools. International Journal of

689 School \& Educational Psychology, 8(2), 119-130.

$690 \quad$ https://doi.org/10.1080/21683603.2019.1694112

691 Maliniak, D., Powers, R., \& Walter, B. F. (2013). The Gender Citation Gap in

692 International Relations. International Organization, 67(4), 889-922.

693 https://doi.org/10.1017/S0020818313000209

694 McGorry, P. D., Hartmann, J. A., Spooner, R., \& Nelson, B. (2018). Beyond the "at risk 695 mental state" concept: Transitioning to transdiagnostic psychiatry. World

696 Psychiatry, 17(2), 133-142. https://doi.org/10.1002/wps.20514 
697 Merikangas, K. R., He, J., Burstein, M., Swendsen, J., Avenevoli, S., Case, B., Georgiades, K., Heaton, L., Swanson, S., \& Olfson, M. (2011). Service Utilization for Lifetime Mental Disorders in U.S. Adolescents: Results of the National Comorbidity Survey-Adolescent Supplement (NCS-A). Journal of the American Academy of Child \& Adolescent Psychiatry, 50(1), 32-45.

703 https://doi.org/10.1016/j.jaac.2010.10.006

Moon, J., Williford, A., \& Mendenhall, A. (2017). Educators' perceptions of youth mental health: Implications for training and the promotion of mental health services in schools. Children and Youth Services Review, 73, 384-391. https://doi.org/10.1016/j.childyouth.2017.01.006

Muris, P., Mayer, B., Freher, N. K., Duncan, S., \& van den Hout, A. (2010). Children's Internal Attributions of Anxiety-Related Physical Symptoms: Age-Related Patterns and the Role of Cognitive Development and Anxiety Sensitivity. Child Psychiatry and Human Development, 41(5), 535-548. https://doi.org/10.1007/s10578-010-0186-1

Obermeyer, Z., Powers, B., Vogeli, C., \& Mullainathan, S. (2019). Dissecting racial bias in an algorithm used to manage the health of populations. Science, 366(6464), 447-453. https://doi.org/10.1126/science.aax2342

O’Brien, D., Harvey, K., Howse, J., Reardon, T., \& Creswell, C. (2016). Barriers to Managing Child and Adolescent Mental Health Problems: A Systematic Review of Primary Care Practitioners' Perceptions. British Journal of General Practice, 66(651), e693-e707. https://doi.org/10.3399/bjgp16X687061 
719 Okulate, G. T., Olayinka, M. O., \& Jones, O. B. E. (2004). Somatic symptoms in

720 depression: Evaluation of their diagnostic weight in an African setting. The

721 British Journal of Psychiatry, 184(5), 422-427.

722 https://doi.org/10.1192/bjp.184.5.422

723 Pina, A. A., \& Silverman, W. K. (2004). Clinical Phenomenology, Somatic Symptoms,

724 and Distress in Hispanic/Latino and European American Youths With Anxiety

725 Disorders. Journal of Clinical Child \& Adolescent Psychology, 33(2), 227-236.

$726 \quad$ https://doi.org/10.1207/s15374424jccp3302_3

727 Quinones-Camacho, L. E. (2018). The Role of Bilingualism and Emotion Regulation on

$728 \quad$ Hispanic Children's Anxiety Development [UC Riverside].

729 https://escholarship.org/uc/item/4hr2w8hb

730 Raymer, D., Weininger, O., \& Hamilton, J. R. (1984). PSYCHOLOGICAL PROBLEMS

731 IN CHILDREN WITH ABDOMINAL PAIN. The Lancet, 323(8374), 439-440.

$732 \quad$ https://doi.org/10.1016/S0140-6736(84)91763-X

733 Reinke, W., Stormont, M., Herman, K., Puri, R., \& Goel, N. (2011). Supporting

734 Children's Mental Health in Schools: Teacher Perceptions of Needs, Roles, and

$735 \quad$ Barriers. School Psychology Quarterly, 26(1), 1-13.

$736 \quad$ https://doi.org/10.1037/a0022714

737 Rushton, J., Bruckman, D., \& Kelleher, K. (2002). Primary Care Referral of Children

738 With Psychosocial Problems. Archives of Pediatrics \& Adolescent Medicine,

739 156(6), 592-598. https://doi.org/10.1001/archpedi.156.6.592 
740 Sakolsky, D., \& Birmaher, B. (2008). Pediatric Anxiety Disorders: Management in

741 Primary Care. Current Opinion in Pediatrics, 20(5), 538.

742 https://doi.org/10.1097/MOP.0b013e32830fe3fa

743 Saps, M., Seshadri, R., Sztainberg, M., Schaffer, G., Marshall, B. M., \& Di Lorenzo, C.

744 (2009). A Prospective School-based Study of Abdominal Pain and Other

745 Common Somatic Complaints in Children. The Journal of Pediatrics, 154(3),

$746 \quad 322-326$. https://doi.org/10.1016/j.jpeds.2008.09.047

747 Shin, S. H. (2005). Need for and actual use of mental health service by adolescents in the

$748 \quad$ child welfare system. Children and Youth Services Review, 27(10), 1071-1083.

$749 \quad$ https://doi.org/10.1016/j.childyouth.2004.12.027

750 Sood, G., \& Laohaprapanon, S. (2018). Predicting Race and Ethnicity From the Sequence

751 of Characters in a Name. ArXiv:1805.02109 [Stat].

752 http://arxiv.org/abs/1805.02109

753 Starfield, B., Gross, E., Wood, M., Pantell, R., Allen, C., Gordon, I. B., Moffatt, P.,

754 Drachman, R., \& Katz, H. (1980). Psychosocial and Psychosomatic Diagnoses in

755 Primary Care of Children. Pediatrics, 66(2), 159-167.

756 Twenge, J. M., Cooper, A. B., Joiner, T. E., Duffy, M. E., \& Binau, S. G. (2019). Age,

757 period, and cohort trends in mood disorder indicators and suicide-related

758 outcomes in a nationally representative dataset, 2005-2017. Journal of Abnormal

759 Psychology, 128(3), 185-199. https://doi.org/10.1037/abn0000410

760 Varela, E., \& Hensley-Maloney, L. (2009). The influence of culture on anxiety in Latino

761 youth: A review. Clinical Child and Family Psychology Review, 12(3), 217-233.

$762 \quad$ https://doi.org/10.1007/s10567-009-0044-5 
763 Varela, E., Sanchez-Sosa, J. J., Biggs, B. K., \& Luis, T. M. (2008). Anxiety Symptoms

764 and Fears in Hispanic and European American Children: Cross-Cultural

765 Measurement Equivalence. Journal of Psychopathology and Behavioral

766 Assessment, 30(2), 132-145. https://doi.org/10.1007/s10862-007-9056-y

767 Waters, A. M., Schilpzand, E., Bell, C., Walker, L. S., \& Baber, K. (2013). Functional

768 Gastrointestinal Symptoms in Children with Anxiety Disorders. Journal of

769 Abnormal Child Psychology, 41(1), 151-163. https://doi.org/10.1007/s10802-012-

$770 \quad 9657-0$

771 Weersing, V. R., Rozenman, M. S., Maher-Bridge, M., \& Campo, J. V. (2012). Anxiety,

772 Depression, and Somatic Distress: Developing a Transdiagnostic Internalizing

773 Toolbox for Pediatric Practice. Cognitive and Behavioral Practice, 19(1), 68-82.

$774 \quad$ https://doi.org/10.1016/j.cbpra.2011.06.002

775 Yatham, S., Sivathasan, S., Yoon, R., da Silva, T. L., \& Ravindran, A. V. (2018).

776 Depression, anxiety, and post-traumatic stress disorder among youth in low and

777 middle income countries: A review of prevalence and treatment interventions.

$778 \quad$ Asian Journal of Psychiatry, 38, 78-91. https://doi.org/10.1016/j.ajp.2017.10.029

779 Yoder, J. R., Whitaker, K., \& Quinn, C. R. (2017). Perceptions of Recidivism Among

780 Incarcerated Youth: The Relationship Between Exposure to Childhood Trauma,

781 Mental Health Status, and the Protective Effect of Mental Health Services in

782 Juvenile Justice Settings. Advances in Social Work, 18(1), 250-269.

$783 \quad$ https://doi.org/10.18060/21305 
784 Yu, K.-H., \& Kohane, I. S. (2019). Framing the challenges of artificial intelligence in

785 medicine. BMJ Quality \& Safety, 28(3), 238-241. https://doi.org/10.1136/bmjqs-

$786 \quad 2018-008551$

787 Zhou, D., Cornblath, E. J., Stiso, J., Teich, E. G., Dworkin, J. D., Blevins, A. S., \&

788 Bassett, D. S. (2020). Gender Diversity Statement and Code Notebook v1.0.

789 Zenodo. https://doi.org/10.5281/zenodo.3672110 


\begin{tabular}{|c|c|c|c|}
\hline Row & $H B N$ & $L A$ & $N Y C$ \\
\hline $\mathrm{N}$ & 1957 & 327 & 102 \\
\hline $\operatorname{Sex}(M / F)$ & $1238 / 719$ & $139 / 188$ & $44 / 58$ \\
\hline Mean age in years (SD, [Range]) & $10.5(3.7,[5,21.9])$ & $8.4(4.1,[2.8,18.6])$ & $10.8(3.5,[4.4,17.6])$ \\
\hline $\begin{array}{l}\text { Mean GI Sum Score (SD, } \\
\text { [Range]) }\end{array}$ & $0.866(1.31,[0,8])$ & $0.361(0.77,[0,4])$ & $0.363(0.77,[0,4])$ \\
\hline Nausea & $0.83 / 0.15 / 0.03$ & $0.94 / 0.06 / 0.01$ & $0.93 / 0.07 / 0$ \\
\hline Vomiting & $0.93 / 0.06 / 0.01$ & $0.99 / 0.01 / 0$ & $0.99 / 0.01 / 0$ \\
\hline Stomachache/Cramps & $0.71 / 0.25 / 0.04$ & $0.85 / 0.13 / 0.02$ & $0.85 / 0.15 / 0$ \\
\hline Constipated & $0.8 / 0.15 / 0.05$ & $0.9 / 0.09 / 0.01$ & $0.89 / 0.08 / 0.03$ \\
\hline Mean SCARED-P (SD, [Range]) & $14.73(12.2,[0,72])$ & $13.71(11.08,[0,67])$ & $12.93(10.98,[0,56])$ \\
\hline $\begin{array}{l}\text { Proportion Meeting SCARED-P } \\
\text { Cutoff }\end{array}$ & 0.19 & 0.15 & 0.13 \\
\hline $\begin{array}{l}\text { Mean SCARED-C (SD, } \\
\text { [Range] })\end{array}$ & $23.21(16.13,[0,80])$ & & \\
\hline $\begin{array}{l}\text { Proportion Meeting SCARED-C } \\
\text { Cutoff }\end{array}$ & 0.4 & & \\
\hline $\begin{array}{l}\text { Proportion w/KSADS Anxiety } \\
\text { Diagnosis }\end{array}$ & 0.32 & & \\
\hline $\begin{array}{l}\text { Median Annual Household } \\
\text { Income }\end{array}$ & $\$ 90,000-99,999$ & $\$ 100,001-\$ 150,000$ & $\$ 150,001-\$ 200,000$ \\
\hline
\end{tabular}

Table 1: Sample information on sex, age, GI symptoms, anxiety symptoms, and median annual household income for all cohorts. 


\begin{tabular}{|c|c|c|c|}
\hline Cohort & Race & $N$ & $P c t$ \\
\hline \multirow[t]{11}{*}{ HBN } & White & 863 & 50.03 \\
\hline & Two or more races & 284 & 16.46 \\
\hline & Black & 276 & 16.00 \\
\hline & Hispanic & 190 & 11.01 \\
\hline & Asian & 51 & 2.96 \\
\hline & Other & 31 & 1.80 \\
\hline & Unknown & 14 & 0.81 \\
\hline & Indian & 11 & 0.64 \\
\hline & Native American & 3 & 0.17 \\
\hline & Alaskan Native & 1 & 0.06 \\
\hline & Native Hawaiian & 1 & 0.06 \\
\hline \multirow[t]{6}{*}{ LA } & White & 125 & 43.10 \\
\hline & Asian & 82 & 28.28 \\
\hline & Two or more races & 40 & 13.79 \\
\hline & Black & 26 & 8.97 \\
\hline & Hispanic & 15 & 5.17 \\
\hline & Native American & 2 & 0.69 \\
\hline \multirow[t]{6}{*}{ NYC } & White & 24 & 26.67 \\
\hline & Asian & 22 & 24.44 \\
\hline & Black & 17 & 18.89 \\
\hline & Two or more races & 12 & 13.33 \\
\hline & Hispanic & 11 & 12.22 \\
\hline & Other & 4 & 4.44 \\
\hline
\end{tabular}

Table 2: Child race in each cohort for all participants with available race data.

Questionnaire items assessing child race differed somewhat across cohorts. While US federal government agencies define Hispanic origin as an ethnicity (US Census Bureau, 2020), not a race, Hispanic was one of the racial categories for which parents could indicate their children belonged in each cohort. 


\begin{tabular}{lccc}
\hline \hline Cohort & Ethnicity & $N$ & Pct \\
\hline HBN & Not Hispanic or Latino & 1149 & 66.19 \\
& Hispanic or Latino & 443 & 25.52 \\
& Decline to Specify & 116 & 6.68 \\
& Unknown & 28 & 1.61 \\
LA & Not Hispanic or Latino & 281 & 87.27 \\
& Hispanic or Latino & 41 & 12.73 \\
NYC & Not Hispanic or Latino & 37 & 84.09 \\
& Hispanic or Latino & 7 & 15.91 \\
\hline \hline
\end{tabular}

Table 3: Child ethnicity in each cohort for all participants with available ethnicity data. Questionnaire items assessing child ethnicity differed somewhat across cohorts. 